\title{
Dispersion of guided modes in two-dimensional split ring lattices
}

\author{
Hansen, Per Lunnemann; Koenderink, A. Femius
}

Published in:

Physical Review B

Link to article, DOI:

10.1103/PhysRevB.90.245416

Publication date:

2014

Document Version

Publisher's PDF, also known as Version of record

Link back to DTU Orbit

Citation (APA):

Hansen, P. L., \& Koenderink, A. F. (2014). Dispersion of guided modes in two-dimensional split ring lattices.

Physical Review B, 90(24), [245416]. https://doi.org/10.1103/PhysRevB.90.245416

\section{General rights}

Copyright and moral rights for the publications made accessible in the public portal are retained by the authors and/or other copyright owners and it is a condition of accessing publications that users recognise and abide by the legal requirements associated with these rights.

- Users may download and print one copy of any publication from the public portal for the purpose of private study or research.

- You may not further distribute the material or use it for any profit-making activity or commercial gain

- You may freely distribute the URL identifying the publication in the public portal

If you believe that this document breaches copyright please contact us providing details, and we will remove access to the work immediately and investigate your claim. 


\title{
Dispersion of guided modes in two-dimensional split ring lattices
}

\author{
Per Lunnemann ${ }^{1}$ and A. Femius Koenderink ${ }^{2, *}$ \\ ${ }^{1}$ DTU Fotonik, Department of Photonics Engineering, Østedsplads 343, DK-2800 Kgs. Lyngby, Denmark \\ ${ }^{2}$ Center for Nanophotonics, FOM Institute AMOLF, Science Park 104, 1098 XG Amsterdam, The Netherlands
}

(Received 15 October 2014; revised manuscript received 1 December 2014; published 10 December 2014)

\begin{abstract}
We present a semianalytical point-dipole method that uses Ewald lattice summation to find the dispersion relation of guided plasmonic and bianisotropic modes in metasurfaces composed of two-dimensional (2D) periodic lattices of arbitrarily strongly scattering magnetoelectric dipole scatterers. This method takes into account all retarded electrodynamic interactions as well as radiation damping self-consistently. As illustration, we analyze the dispersion of plasmon nanorod lattices, and of 2D split ring resonator lattices. Plasmon nanorod lattices support transverse and longitudinal in-plane electric modes. Scatterers that have an in-plane electric and out-of-plane magnetic polarizability, but without intrinsic magnetoelectric coupling, result in two bands that are mixtures of the bands of electric-only and magnetic-only lattices. Thereby, bianisotropy through mutual coupling, in absence of building-block bianisotropy, is evident. Once strong bianisotropy is included in each building block, the Bloch modes become even more strongly magnetoelectric. Our results are important to understand spatial dispersion and bianisotropy of metasurface and metamaterial designs.
\end{abstract}

\section{INTRODUCTION}

Periodic structures of scatterers have a long-standing history in photonics, traditionally in the guise of diffraction gratings [1-3] and photonic crystals [4,5], and more recently in the context of plasmonics, metamaterials, and metasurfaces [6-8]. In plasmonics with noble-metal particles that support localized resonances, periodic chains of particles with subdiffraction pitch were already proposed in 1998 [9] and demonstrated in 2003 [10] as potential candidates for guiding signals in a deep subwavelength fashion through near-field dipole-dipole interaction [9-15]. While transport in these systems is very lossy, the exact formalism to describe the guiding mechanism in presence of long-range retarded dipole-dipole interactions has remained a topic of ongoing work $[9,11-13,16]$. This is in part due to the associated mathematical intricacies [17] and in part to the fact that transport along chains of Lorentzian dipole resonators transcends plasmonics in relevance. Twodimensionally periodic systems of plasmon particles have traditionally been studied in the case of diffractive lattices, in which case grating anomalies hybridize with localized surface plasmon resonances to give very sharp spectral features [18-21]. These features have been pursued for field-enhanced spectroscopies [22,23], sensing [24,25], as well as improved solid-state light sources [26-28]. Since the advent of 2D metamaterial arrays, the response of subdiffraction pitch lattices of resonant scatterers has gained significantly in relevance [7,8,29].

While experimental studies of two-dimensional (2D) metamaterials and metasurfaces usually probe transmission and reflection for some definite incident polarization and wave vector [30-32], the fundamental underlying property of a lattice must be its dispersion relation or band structure, which summarizes the existence of guided as well as leaky modes. The spectrum of leaky modes supported by a lattice of split rings, for instance, would explain the origin of angle-dependent transmission and reflection features [33], and would form an excellent basis to understand spatial dispersion in attributed effective material constants [34-36]. Complementary to the leaky modes, the guided mode structure would also be relevant, for instance for the proposed "lasing spaser" [28] where a 2D metamaterial lattice is immersed in a gain medium, or when coupling a localized fluorescent source to a lattice in the near field. In this case, the modes subject to most gain, or the modes with strongest coupling to the source, need not correspond to resonances identified in normal incidence scattering experiments. Rather, any guided modes supported by the lattice could be excited in any experiment that does not a priori restrict or impose parallel wave vector. An understanding of the band structure of 2D lattices of magnetic and electric resonant scatterers is therefore important for metasurface research.

Previously, modal band structures of a 2D lattice of electric dipolar spherical scatterers have been theoretically treated [16,37], while only the leaky modes of split ring resonators (SRR) lattices have so far been assessed through transmission calculations and compared to experiments [30,31,33]. In this paper, we present a method to calculate band structures for arbitrary lattices of arbitrary magnetoelectric dipolar scatterers, and illustrate its properties for simple lattices of plasmon rods, as well as idealized split rings.

\section{LATTICE RESPONSE}

We consider a 2D lattice consisting of arbitrary magnetoelectric point scatterers in the dipole approximation, however, without making any electrostatic approximation. Each particle is described by a polarizability tensor $\stackrel{\leftrightarrow}{\alpha}$ that relates the induced electric and magnetic dipole moment $\boldsymbol{p}$ and $\boldsymbol{m}$ to a driving electric and magnetic field $\boldsymbol{E}$ and $\boldsymbol{H}$ according to [38,39]

\footnotetext{
*fkoenderink@amolf.nl
}

$$
\left(\begin{array}{c}
\boldsymbol{p} \\
\boldsymbol{m}
\end{array}\right)=\overleftrightarrow{\alpha}\left(\begin{array}{c}
\boldsymbol{E} \\
\boldsymbol{H}
\end{array}\right)
$$


The magnetoelectric polarizability may be decomposed as

$$
\overleftrightarrow{\alpha}=\left(\begin{array}{ll}
\overleftrightarrow{\alpha}_{E E} & \stackrel{\leftrightarrow}{\alpha}_{E H} \\
\stackrel{\leftrightarrow}{\alpha}_{H E} & \stackrel{\leftrightarrow}{\alpha}_{H H}
\end{array}\right)
$$

where $\stackrel{\leftrightarrow}{\alpha}_{E E}$ is the $3 \times 3$ electric polarizability tensor that quantifies the induced electric dipole moment in response to an electric field. Similarly, $\stackrel{\leftrightarrow}{\alpha}_{H H}$ describes the magnetic polarizability that quantifies the induced magnetic dipole in response to a magnetic driving field. Finally, $\stackrel{\leftrightarrow}{\alpha}_{E H}=$ $-\stackrel{\leftrightarrow}{\alpha}_{H E}^{T}$ denotes the magnetoelectric coupling that describes the induced electric dipole moment in response to a magnetic field and vice versa. This element controls the bianisotropy [38] of the medium giving rise to chiral extinction under oblique incidence $[39,40]$. We shall denote $\stackrel{\leftrightarrow}{\alpha}$ the bare polarizability since it describes the induced dipole moments in the absence of neighboring point scatterers. $\stackrel{\leftrightarrow}{\alpha}$ is subject to reciprocity and energy conservation constraints discussed in Refs. [39,41]. We construct the electrodynamically consistent polarizability of a single scatterer, bound by the optical theorem, by addition of radiation damping

$$
\stackrel{\leftrightarrow}{\alpha}^{-1}=\stackrel{\leftrightarrow}{\alpha}_{0}^{-1}-\frac{2}{3} k^{3} i \mathbb{I}
$$

to an electrostatic bare polarizability tensor $\stackrel{\leftrightarrow}{\alpha}_{0}$ which can for instance be derived from an inductor-capacitance (LC) circuit model. Here, $k$ denotes the wave number, $\mathbb{I}$ is the six-dimensional identity tensor, and $(\ldots)^{-1}$ denotes matrix inversion. In this work, we will illustrate band-structure calculations by considering a specific group of scatterers representative of plasmon rods and of many metamaterial scatterers like SRR. In particular, we assume the only available responses to be electric along the $x$ direction (along the bar, or split, see Fig. 1) and/or magnetic along the $z$ direction (direction through the SRR loop), setting all other tensor elements to zero. That is,

$$
\overleftrightarrow{\alpha}_{0}=\mathcal{L}(\omega)\left(\begin{array}{ccccc}
\eta_{E} & 0 & \ldots & 0 & i \eta_{C} \\
0 & 0 & & & 0 \\
\vdots & & \ddots & & \vdots \\
0 & & & 0 & 0 \\
-i \eta_{C} & 0 & \ldots & 0 & \eta_{H}
\end{array}\right)
$$

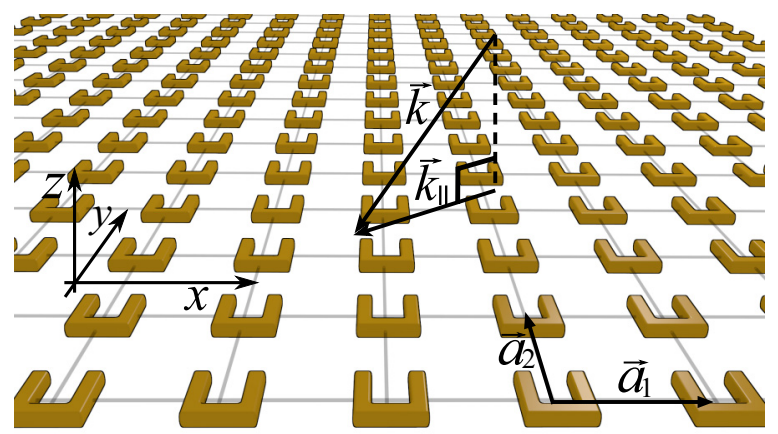

FIG. 1. (Color online) Illustration of the considered lattice, here sketched for split ring resonators, with a plane wave incident with an in-plane wave vector $k_{\|}$. where $\mathcal{L}(\omega)$ is a Lorentzian prefactor

$$
\mathcal{L}(\omega)=V \frac{\omega_{0}^{2}}{\omega_{0}^{2}-\omega^{2}-i \omega \gamma},
$$

typical for a plasmon resonance or LC circuit model, where $V$ is the physical volume of the scatterer, $\gamma$ is the damping rate due to Ohmic losses, $\omega_{0}$ denotes the resonance frequency, and $\eta_{E, H, C}$ are real dimensionless parameters that for LC circuits can be calculated from geometry. Recalling that the extinction cross section of a simple scatterer with scalar polarizability $\alpha$ is $\sigma_{\text {ext }}=4 \pi k \operatorname{Im}(\alpha)$, we note that for tensorial polarizability, the extinction cross section varies with incidence condition, but is always a linear combination of the imaginary part of the tensor eigenvalues $\alpha_{1}$ and $\alpha_{2}$ of Eq. (3). The corresponding eigenvectors may possess a magnetoelectric character, having both a component along $p_{x}$ and $m_{z}$ through intrinsic magnetoelectric coupling when $\eta_{C} \neq 0$ [39]. Eigenvectors with a phase-offset between $p_{x}$ and $m_{z}$ results in a "pseudochirality," i.e., a handedness-dependent extinction for some incidence angles. We note that the maximum value $\eta_{C}$ can attain is $\sqrt{\eta_{E} \eta_{H}}$, at which point one of the two eigenpolarizabilities reaches 0 and the other reaches $\mathcal{L}(\omega)\left[\eta_{E}+\eta_{H}\right]$. For LC-circuit scatterers, maximum cross coupling is the norm, while removing cross coupling is a challenge.

Based upon the discrete dipole approximation method (DDA) [42], the optical response of 2D periodic lattices of electric polarizabilities was didactically reviewed by de Abajo [43] and extended to the full magnetoelectric case in Refs. [30,44]. For consistency, we recapitulate the main findings. Consider a 2D periodic lattice of point scatterers placed at $\boldsymbol{R}_{m n}=m \boldsymbol{a}_{1}+n \boldsymbol{a}_{2}$ (where $m$ and $n$ are integers, and $\boldsymbol{a}_{1,2}$ are the real-space basis vectors, see 1). The response of a particle at position $\boldsymbol{R}_{m n}$ is self-consistently set by the incident field, plus the field of all other dipoles in the lattice according to [43]

$$
\begin{aligned}
\left(\begin{array}{c}
\boldsymbol{p}_{m n} \\
\boldsymbol{m}_{m n}
\end{array}\right)= & \stackrel{\leftrightarrow}{\alpha}\left[\left(\begin{array}{c}
\boldsymbol{E}_{\mathrm{in}}\left(\boldsymbol{R}_{m n}\right) \\
\boldsymbol{H}_{\mathrm{in}}\left(\boldsymbol{R}_{m n}\right)
\end{array}\right)\right. \\
& \left.+\sum_{m^{\prime} \neq m, n^{\prime} \neq n} \stackrel{\leftrightarrow}{G} 0\left(\boldsymbol{R}_{m n}-\boldsymbol{R}_{m^{\prime} n^{\prime}}\right)\left(\begin{array}{c}
\boldsymbol{p}_{m^{\prime} n^{\prime}} \\
\boldsymbol{m}_{m^{\prime} n^{\prime}}
\end{array}\right)\right]
\end{aligned}
$$

where $\stackrel{\leftrightarrow}{G}^{0}\left(\boldsymbol{R}_{m n}-\boldsymbol{R}_{m^{\prime} n^{\prime}}\right)$ is the $6 \times 6$ dyadic Green's function of the medium surrounding the lattice. Plane-wave incidence with parallel wave vector $\boldsymbol{k}_{\|}$allows a Bloch wave form $\left(\boldsymbol{p}_{m n}, \boldsymbol{m}_{m n}\right)^{T}=e^{i \boldsymbol{k}_{\|} \cdot \boldsymbol{R}_{m n}}\left(\boldsymbol{p}_{00}, \boldsymbol{m}_{00}\right)^{T}$ to obtain

$$
\left(\begin{array}{c}
\boldsymbol{p}_{00} \\
\boldsymbol{m}_{00}
\end{array}\right)=\left[\stackrel{\leftrightarrow}{\alpha}-1-\stackrel{\leftrightarrow}{G}\left(\boldsymbol{k}_{\|}, 0\right)\right]^{-1}\left(\begin{array}{c}
\boldsymbol{E}_{\mathrm{in}}\left(\boldsymbol{R}_{00}\right) \\
\boldsymbol{H}_{\mathrm{in}}\left(\boldsymbol{R}_{00}\right)
\end{array}\right) .
$$

Here, $\stackrel{\leftrightarrow}{G}\left(\boldsymbol{k}_{\|}, 0\right)$ is a summation of the dyadic Green's function $\stackrel{\leftrightarrow}{G}^{0}$ over all positions in the lattice barring the origin:

$$
\stackrel{\leftrightarrow}{\mathcal{G}}^{\neq}\left(\boldsymbol{k}_{\|}, \boldsymbol{r}\right)=\sum_{m \neq 0, n \neq 0} \stackrel{\leftrightarrow}{G}^{0}\left(\boldsymbol{R}_{m n}-\boldsymbol{r}\right) e^{i \boldsymbol{k}_{\|} \cdot \boldsymbol{R}_{m n}} .
$$

In this work, we take the surrounding medium, that defines $\stackrel{\leftrightarrow}{G}^{0}$, to be homogeneous. Implementation of the sum of $\stackrel{\leftrightarrow}{G}^{0}$ was carried out using the Ewald lattice summation technique [17], that consists of splitting a poorly convergent sum, such as 
Eq. (8), into two exponentially convergent sums as summarized in Refs. [30,44]. The same techniques, can be extended to lattices in stratified dielectric systems, complex unit cells, and stacks of lattices [44].

The factor $\left[\stackrel{\leftrightarrow}{\alpha}^{-1}-\stackrel{\leftrightarrow}{\mathcal{G}}\left(\boldsymbol{k}_{\|}, 0\right)\right]^{-1}$ in Eq. (7) is identified as an effective polarizability tensor $\stackrel{\leftrightarrow}{\alpha}_{\text {eff }}$ of the scatterer, renormalized by the scattering from all other lattice sites. In a lossless system, the Bloch wave dispersion would correspond to those frequencies for which $\operatorname{det}\left(\stackrel{\leftrightarrow}{\alpha}^{-1}-\stackrel{\leftrightarrow}{\mathcal{G}}^{\neq}\right)=$ 0 or, equivalently, those frequencies for which $\alpha_{\text {eff }}$ has a pole. These two-dimensional lattices in fact have radiative (if $k_{\|} \leqslant \omega / c$ ) and Ohmic loss. For lossy systems, a real dispersion relation is not defined, and one should in principle seek either complex wave-vector-real-frequency solutions, or conversely complex-frequency-real-wave-vector solutions for which $\operatorname{det}\left(\stackrel{\leftrightarrow}{\alpha}^{-1}-\stackrel{\leftrightarrow}{\mathcal{G}}^{\neq}\right)=0$ as first noted by Barker and Loudon $[11,45]$. We simply evaluate $\stackrel{\leftrightarrow}{\alpha}^{-1}-\stackrel{\leftrightarrow}{\mathcal{G}}^{\neq}$for real $\omega$ and real $k_{\|}$. In particular, we consider the imaginary part of the eigenvalues of $\left[\stackrel{\leftrightarrow}{\alpha}^{-1}-\stackrel{\leftrightarrow}{\mathcal{G}}^{\neq}\right]^{-1}$ as they directly relate to extinction. Distinct bands emerge, the width of which we identify as the damping rate [37] due to both Ohmic and radiation damping. For the effectively $2 \times 2$ form of the single-particle polarizability that we use in this paper [Eq. (4)], for each $\left(\omega, k_{\|}\right)$point we obtain at most two nontrivial eigenvectors and eigenvalues. To separate the dispersion in bands, we group eigenvalues by continuity of the projection of the corresponding eigenvectors with the eigenvectors of neighboring $\left(\omega, k_{\|}\right)$points.

\section{RESULTS}

As illustration, we consider four types of scatterers, starting with plasmon rods and culminating at a realistic description for split rings. It has been experimentally demonstrated that SRRs are well described with a dipolar polarizability as in Eqs. (4) and (3) provided one takes bianisotropy $\eta_{C}$ at the upper limit $\sqrt{\eta_{E} \eta_{H}}[30,39,40]$. To help understand what effect magnetoelectric coupling has, we consider three subcases prior to analyzing the SRR lattice with maximum intrinsic coupling. These consist of (1) plasmon rods along $x$ (only $\eta_{E} \neq 0$ ), (2) magnetic dipolar antennas along $z$ (only $\eta_{H} \neq 0$ ), and (3) uncoupled SRRs without bianisotropy (setting $\eta_{E}=\eta_{H}=1$ and $\eta_{C}=0$ ). Throughout the paper, we use parameter values as stated in Table I. To more clearly resolve the modes, we take an Ohmic damping rate $\gamma \sim 100$ times less than that of gold [46]. The parameters yield an extinction cross section per scatterer of $0.2-0.3 \mu \mathrm{m}^{2}$, comparable to measured values $[30,47]$.

TABLE I. Chosen parameter values.

\begin{tabular}{lcl}
\hline \hline Parameter & Value & \multicolumn{1}{c}{ Description } \\
\hline$V$ & $(80 \mathrm{~nm})^{3}$ & Physical volume of scatterer \\
$\gamma$ & $1 \times 10^{12} \mathrm{~s}^{-1}$ & Damping rate \\
$a_{1}=a_{2}$ & $300 \mathrm{~nm}$ & Lattice constant \\
$\omega_{0}$ & $2 \pi c / 1.5 \mu \mathrm{m}$ & Resonance frequency \\
\hline \hline
\end{tabular}

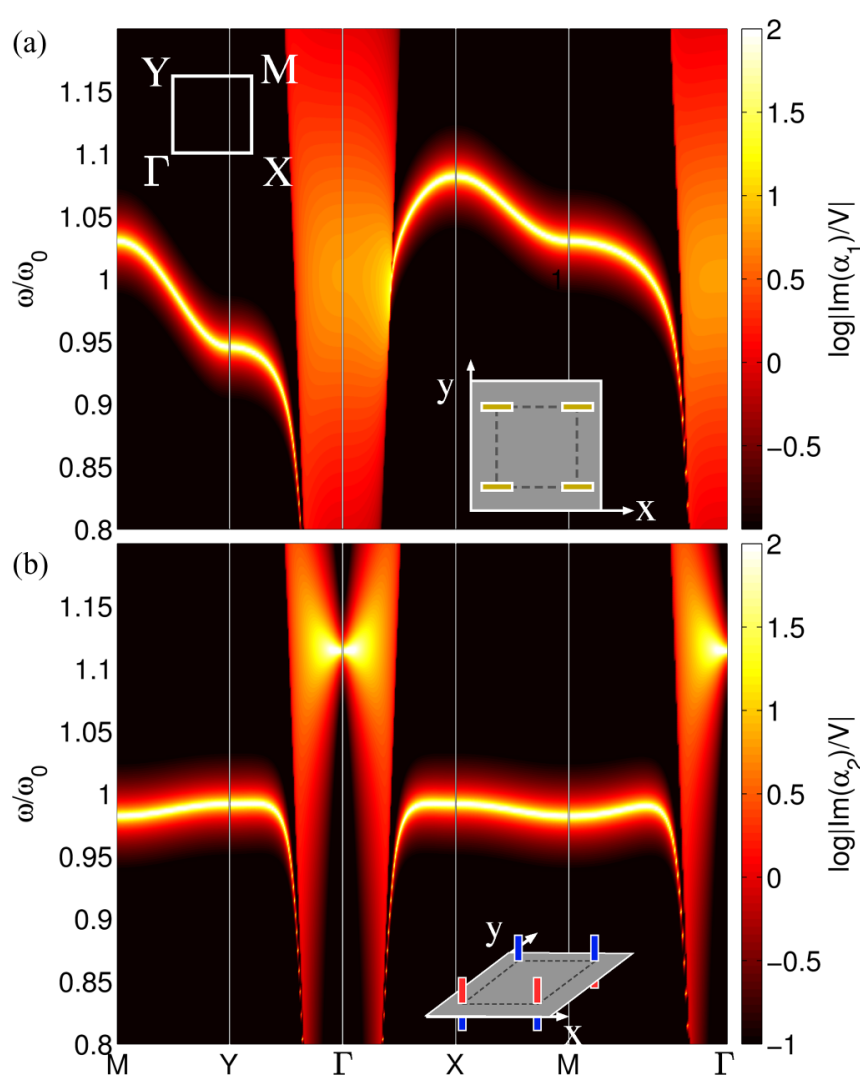

FIG. 2. (Color online) Calculated effective polarizability $\ln [\operatorname{Im}(\alpha) / V]$ as a function of $k_{\|}$and $\omega$ for (a) an in-plane bar-type electric structure as illustrated in the inset with $\eta_{H}=\eta_{C}=0$ and $\eta_{E}=1$ in Eq. (4) and (b) a transverse bar-type magnetic structure as illustrated in the inset with with $\eta_{E}=\eta_{C}=0$ and $\eta_{H}=1$ in Eq. (4).

\section{A. Scalar anisotropic scatterers}

We first consider plasmon rods with bare polarizability given by Eq. (4) and setting $\eta_{H}=\eta_{C}=0$ and $\eta_{E}=$ 1. In Fig. 2(a), we present the calculated dispersion diagram sweeping $\left(k_{x}, k_{y}\right)$ through the reduced Brillouin zone along the following path: $(\pi / d, \pi / d) \rightarrow(0, \pi / d) \rightarrow$ $(0,0) \rightarrow(\pi / d, 0) \rightarrow(\pi / d, \pi / d) \rightarrow(0,0)$ also denoted $\mathrm{M} \rightarrow$ $\mathrm{Y} \rightarrow \Gamma \rightarrow \mathrm{X} \rightarrow \mathrm{M} \rightarrow \Gamma$. The discontinuity on each side of $\Gamma$ indicates the light line. The sharp resonances below the light line are guided modes of the lattice. Two distinct modes are observed on either side of the $\Gamma$ point. Within the domain $\Gamma \rightarrow \mathrm{Y}$, the dipole phase is constant along $\hat{\boldsymbol{x}}$ and varies along $\hat{\boldsymbol{y}}$, transverse to the dipole moment orientation. Thus, this mode is a TIE mode, with dipole moments perpendicular to $k_{\|}$. The heads to tail arrangement along $\hat{\boldsymbol{x}}$ and alternating direction when going along $\hat{\boldsymbol{y}}$ results in a red-shift of the resonance as has previously been explained using simple hybridization models for electrostatic 1D and 2D systems [11-13,48,49]. In such a hybridization model, resonance shifts of coupled dipoles can be understood from considering the quasistatic interaction energy $U$ between two dipoles $\boldsymbol{p}_{1}$ and $\boldsymbol{p}_{1}$ separated by a vector $\boldsymbol{r}$ :

$$
U \propto \frac{\boldsymbol{p}_{1} \cdot \boldsymbol{p}_{2}-3\left[\boldsymbol{p}_{1} \cdot \hat{\boldsymbol{r}}\right]\left[\boldsymbol{p}_{2} \cdot \hat{\boldsymbol{r}}\right]}{r^{3}} .
$$


Accordingly, longitudinal coupling of parallel (antiparallel) dipoles leads to red-shifts (blue-shifts), while for transversely coupled dipoles the coupling strength is reduced and opposite in sign. As caveat we note that such hybridization models strictly apply only in electrostatics, whereas here we treat retarded interactions between lossy, resonant dipoles.

Analogously, within the domain $\Gamma \rightarrow \mathrm{X}$ all dipoles point along the wave vector, and the mode is therefore LIE [37]. In this configuration, the head-to-head arrangement along $\hat{\boldsymbol{x}}$ and the fixed direction along $\hat{\boldsymbol{y}}$ results in a blue-shift. For the regions $\mathrm{Y} \rightarrow \mathrm{M}$ and $\mathrm{X} \rightarrow \mathrm{M}$, the mode possesses a mixed transverse and longitudinal character. For wave vectors within the light cone, we notice a faint resonance. This resonance is very broad due to radiation damping, and is the resonance that is probed in far-field transmission spectra [30-32]. Compared to the single-particle radiative linewidth, the collective resonance linewidth is broader by more than an order of magnitude. This collective superradiant damping effect has been observed experimentally in density-dependent studies of transmission at normal incidence for 2D lattices [31]. Since the time-averaged far-field flux from a single dipole pointing along $\hat{\boldsymbol{x}}$ is proportional to $\sin ^{2}(\theta)$ with $\theta$ being the azimuthal angle along $x$ [50], scattering out of the lattice plane is strong for in-plane modes. For longitudinal modes, this damping monotonically reduces with increasing $k_{\|}$as dipoles do not radiate along their axis $[11,13]$. For transverse modes, the radiation damping is constant or increases with $k_{\|}$when approaching the light line. Finally, the modes far below the light line have a constant width comparable to the single-particle Ohmic damping rate $\gamma$. However, very close to the light line, the damping rates drop well below the Ohmic damping rate indicating that the modes near the light line are very weakly confined and have almost no mode overlap with the metal scatterers. As is the case for, e.g., a thin dielectric slab in a symmetric host environment, even a plane of weakly polarizable particles binds a guided mode, however, with a very large fraction of its energy density in air. We note the strong similarities to the calculated dispersion of $1 \mathrm{D}$ as well as 2D lattices of spherical electric scatterers [11,13,37].

We now turn to the case of out-of-plane magnetic scatterers with bare polarizability set by $\eta_{E}=\eta_{C}=0$ and $\eta_{H}=1$ in Eq. (4). This is a hypothetical case as magnetic scatterers are not readily available. Yet, very high index dielectric spheres and spheroids have magnetic dipole resonances, so that realizations could be envisioned [51]. In Fig. 2(b), we present the calculated effective polarizability. The dispersion is symmetric about $\Gamma$ owing to the rotational symmetry of this lattice. Obviously, the observed mode is a TM mode. As opposed to the TIE and LIE modes in Fig. 2, we can clearly resolve a resonance above the light line. For a single transverse dipole, the radiated intensity perpendicular to the lattice is zero, while radiation in the plane is strong [50]. Hence, for the array at $k_{\|}=0$ there is no radiative loss. The increasing broadening when going from $k_{\|}=0$ to the light line, was also claimed for $1 \mathrm{D}$ chains of plasmonic particles $[11,13]$. As the wave vector sweeps to the light line, the radiative loss, which by momentum conservation has the same in-plane wave vector, has an increasingly good overlap with the single dipole radiation pattern, thereby causing the radiative loss to increase from 0 at $k_{\|}=0$ to large values.
Comparing Figs. 2(a) and 2(b), the TIE and TM modes are seen to converge asymptotically to the light line, while the LIE mode crosses the light line. For the transverse modes, all dipoles are perpendicular to the propagation vector and therefore couple strongly to free photons with an anticrossing as a result. For the LIE mode, all dipoles are parallel to the wave vector and thus hindered from coupling to the far field. Consequently, no anticrossing is observed in good agreement with previous results on $1 \mathrm{D}$ and $2 \mathrm{D}$ arrays of scatterers $[11,13,37]$. Finally, we note that the modes of the out-of-plane magnetic antennas are much less dispersive than those of the electric in-plane antennas showing an almost flat band between Y-M and X-M. Inspecting the interaction energy in the magnetostatic equivalent of Eq. (9), we note that for the TM mode near Y (X) a blue-shift is induced from adjacent parallel dipoles along $\hat{\boldsymbol{x}}(\hat{\boldsymbol{y}})$ while a red-shift of equal magnitude is induced from adjacent antiparallel dipoles along $\hat{\boldsymbol{y}}(\hat{\boldsymbol{x}})$, leading to net cancellation of the hybridization energies. This cancellation of nearest-neighbor contributions holds for any periodicity, i.e., also for retarded interactions. To the contrary, for the electric in-plane antennas near $\mathrm{Y}(\mathrm{X})$, the heads-to-tail arrangement along $\hat{\boldsymbol{x}}(\hat{\boldsymbol{y}})$ and antiparallel arrangement along $\hat{\boldsymbol{y}}(\hat{\boldsymbol{x}})$ both contribute with a red-shift (blue-shift). Hence, any path connecting $\mathrm{Y}$ and $\mathrm{X}$ exhibits a larger variation in frequencies compared to the TM mode.

\section{B. Split ring resonators}

We now turn our attention to SRR-type scatterers. We describe the per-building block magnetoelectric coupling by the parameter $\eta_{C}$ in Eq. (4). Energy conservation dictates that $\eta_{C}$ is bound by $\left|\eta_{C}\right| \leqslant \sqrt{\eta_{E} \eta_{H}}$ [39], where equality holds for a truly planar scatterer described as a single resonant circuit. It has been demonstrated experimentally, and by full-wave simulations [30,39,40,52], that real SRRs indeed possess a coupling strength $\eta_{C}$ close to the upper limit $\sqrt{\eta_{E} \eta_{H}}$. We note that, for more complicated scatterers though, i.e. nested split rings, lower cross couplings can occur [40,52]. For clarity, we shall first consider the two cases of absent and partial coupling between the magnetic and electric dipole by setting $\eta_{C}=0$ and $\eta_{C}=0.5 \sqrt{\eta_{E} \eta_{H}}$, respectively, in Eq. (4) before finally considering the realistic case with full cross coupling $\eta_{C}=\sqrt{\eta_{E} \eta_{H}}$. The calculated dispersion diagrams are presented in Fig. 3.

\section{SRR without cross coupling (anisotropic)}

We start by considering the case of no electromagnetic cross coupling, $\eta_{C}=0$ and $\eta_{E}=\eta_{H}=1$. In this case, for each $\left(k_{\|}, \omega\right)$ pair, two nontrivial eigenvalues exist. In Fig. 3(a), the calculated sum of imaginary parts of eigenvalues $\operatorname{Im} \alpha_{1}+\operatorname{Im} \alpha_{2}$ is plotted. We immediately identify that the dispersion diagram resembles the superposition of those in Fig. 2 for the purely electric and purely magnetic objects. One mode traces the in-plane mode in Fig. 2(a), while we observe some differences between the other mode and the TM mode in Fig. 2(b), especially for the region $\mathrm{M}-\mathrm{Y}-\Gamma$. These differences arise from interparticle coupling between electric dipoles, magnetic dipoles, and between electric and magnetic dipoles. 

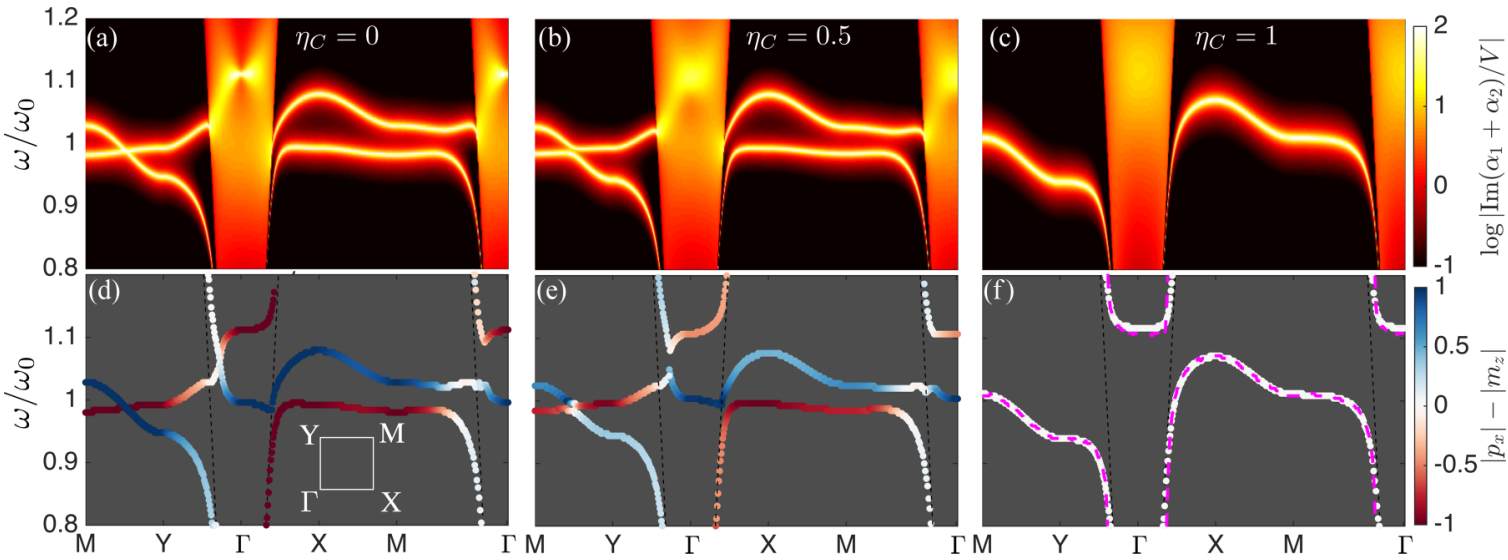

FIG. 3. (Color online) (a)-(c) Calculated sum of eigenvalues $\ln \left[\operatorname{Im}\left(\alpha_{1}+\alpha_{2}\right) / V\right]$ as a function of wave vector $k_{\|}$and normalized frequency $\omega / \omega_{0}$ for a SRR lattice with (a) cross coupling $\eta_{C}=0$ and (b) $\eta_{C}=0.5$, and (c) $\eta_{C}=1$. (d)-(f) Calculated mixing ratio $\zeta=\left|p_{x}\right|-\left|m_{z}\right|$ of the eigenvectors for the extracted bands. Blue corresponds to a pure electric dipole, while red corresponds to pure magnetic dipole. White is a balanced mix of magnetic and electric dipoles. Dashed black lines indicate the light lines. Magenta dashed line in (f) indicates the sum of frequency shifts $\Delta \omega_{E}\left(k_{\|}\right)+\Delta \omega_{H}\left(k_{\|}\right)+\omega_{0}$ of the two modes in (d).

To clarify how Figs. 3(a) and 2(b) (TM mode) differ, we define a electromagnetic mixing ratio as

$$
\zeta_{j}=\left|p_{j, x}\right|-\left|m_{j, z}\right|,
$$

where $p_{j, x}\left(m_{j, z}\right)$ is the electric $x$ (magnetic $z$ ) component of the $j$ th normalized eigenvector, i.e., $\sqrt{\left|p_{j, x}\right|^{2}+\left|m_{j, z}\right|^{2}}=1$. For $\zeta=-1$ the mode is purely TM, while for $\zeta=1$ the mode is purely in-plane electric. The mixing ratio of the bands in Fig. 3(a) is presented in Fig. 3(d). Generally, the two modes are clearly identifiable as strongly electric resp. magnetic, as expected if the dispersion were that of an uncoupled electric lattice and magnetic lattice. In fact, the modes are purely inplane electric, respectively TM, at all the symmetry points M, $\mathrm{Y}, \Gamma$, and $\mathrm{X}$. A strongly mixed character occurs close to the light line, in particular midway $\Gamma$ and $Y$, and at the light line crossing between $\Gamma$ and $\mathrm{M}$, commensurate with the fact that there the mode must match a plane wave propagating in the array plane, which carries both out of plane $H$ and in plane $E$.

Before we introduce cross coupling $\eta_{C}$, we consider the hybridization interaction between magnetic and electric dipoles. In Fig. 4, we illustrate the spatial distribution of
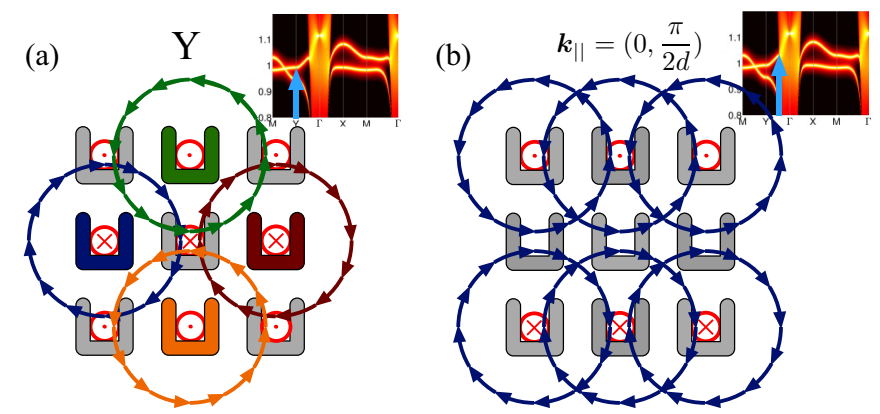

FIG. 4. (Color online) Illustration of the distribution of magnetic dipoles and their associated electric fields in a SRR lattice with $\eta_{C}=0$ for wave vectors at (a) $\mathrm{Y}$ and (b) $\mathrm{Y} / 2$ as indicated by a blue arrow in the dispersion map inset. Red circles with a dot (cross) indicate a magnetic dipole pointing out of (into) the paper. magnetic dipoles and their associated electric fields at the point in $k_{\|}$space $\mathrm{Y}=(0, \pi / 2 d)$ and the point midway between $\Gamma$ and $Y$, denoted as $Y / 2$. Considering the field lines at Y, depicted in Fig. 4(a), at the location of the central SRR the electric fields from adjacent magnetic moments cancel. We therefore conclude that at $\mathrm{Y}$ the lattices of magnetic and electric dipoles are essentially decoupled, in good agreement with the unit value of the mixing ratio in Fig. 3(d). A similar analysis holds for the $\mathrm{X}$ point. Considering the point $\mathrm{Y} / 2$ in Fig. 4(b), the electric field lines of magnetic dipoles adjacent to a central site add up along $\hat{\boldsymbol{x}}$ and from this we infer that the TIE and TM modes strongly mix at $\mathrm{Y} / 2$. This is in contrast to the point $\mathrm{X} / 2$, where the field lines add up along $\hat{\boldsymbol{y}}$, along which the SRR is not polarizable. Hence, no dipole moment is induced and one mode is therefore purely TM, while the other is purely electric in nature. The same results for the absence/presence of magnetoelectric cross coupling is obtained by starting the hybridization analysis from magnetic fields due to in-plane electric dipoles rather than vice versa, as expected from reciprocity.

To conclude, even if one starts with building blocks that have no magnetoelectric coupling, once placed in a dense lattice, the collective modes have mixed electric and magnetic character except at symmetry points.

\section{SRR with cross coupling (bianisotropic)}

Most realized metamaterial lattices will consist of building blocks possessing an intrinsic magnetoelectric coupling that couples the excitation of electric and magnetic dipoles in a single building block according to a definite amplitude and phase relation. We consider partial electromagnetic cross coupling setting $\eta_{C}=0.5$ and $\eta_{E}=\eta_{H}=1$ in Fig. 3(b). Comparing with the case $\eta_{C}=0$ in Fig. 3(a), we immediately see a close resemblance, apart from a clearly resolved anticrossing midway between $\mathrm{M}$ and $\mathrm{Y}$. The calculated mixing ratio [see Fig. 3(e)] evidences that the modes are no longer purely electric or purely magnetic at any of the symmetry points. Near the anticrossing, the mixing ratio becomes 0 implying that the two modes carry equal electric and magnetic content. The 
associated complex phase difference defined as

$$
\Delta \phi_{j}=\arg p_{j, x}-\arg m_{j, z}
$$

is, at the anticrossing point, found to be $\Delta \phi_{1}=\pi / 2$ for the lower band and $\Delta \phi_{2}=-\pi / 2$ for the upper band. This distinct phase difference between the two anticrossing bands signifies that one solution has the electric dipole a quarter cycle in advance of the magnetic one, while for the other solution the electric dipole lags the magnetic dipole by a quarter cycle. This distinction stems from the physics intrinsic to the single bianisotropic scatterer, wherein the polarizability tensor of a cross-coupled scatterer has two distinct eigenvalues, corresponding to high, respectively low, scattering strength, with eigensolution corresponding to either an advanced or lagging electric dipole relative to the magnetic response. In LC-circuit terms, a strong difference in response to a driving field with either an advance or lag can be understood by noting that in the driving $E_{x} d+i \omega H_{z} A$ ( $d$ the capacitor gap, $A$ the loop area) the electric term driving the capacitor and the electromotive force due to a changing flux, either add or cancel depending on phase. In a scattering experiment, this results in a strongly handed response under oblique incidence since oblique incidence circular polarization carries a phase difference between $E_{x}$ and $H_{z}$ [30,40]. Returning to the physics of the lattice, given the Bloch wave vector $k_{\|}=(\mathrm{M}+\mathrm{Y}) / 2$, one can explicitly calculate the fields exerted on a central dipole by all its neighbors. We indeed find that when $p_{x}=i m_{z}$, respectively $p_{x}=-i m_{z}$, the overlap of the fields a central dipole scatterer receives from its neighbors in the lattice is very different in strength (addition resp. cancellation in terms of $\left.E_{x} d+i \omega H_{z} A\right)$.

Finally, we consider the maximally coupled case $\eta_{C}=$ $\eta_{E}=\eta_{H}=1$, presented in Figs. 3(c) and 3(f). The most remarkable aspect is that one of the two bands vanishes, leaving only one band. That this must happen is easily understood by noting that at maximum cross coupling $\eta_{C}=\sqrt{\eta_{E} \eta_{H}}$, one of the two eigenvalues of the single-object polarizability tensor vanishes. In other words, a maximally cross-coupled SRR has just one mode of oscillation and not two, in which furthermore the relative phase and amplitude of $p$ and $m$ are locked. A didactic example is a LC circuit, which has just one resonant mode of oscillation where the same circulating charge gives rise to both $p_{x}$ and $m_{z}$ in a fixed phase and amplitude ratio. This intuition for the LC circuit is only reconcilable with a $2 \times 2$ polarizability tensor if one eigenvalue vanishes. By extension, this also means that a lattice of maximally cross-coupled scatterers presents only one band, not two. A remarkable observation is that, beyond the light line, the band structure is similar to the primarily in-plane mode of the lattice with $\eta_{C}=0$, seen in Figs. 3(a) and 3(d). However, this similarity only holds for the $\left(\omega, k_{\|}\right)$relation but not for the associated eigenfunctions since the eigenfunctions necessarily show equally strong $p$ and $m$ resulting in $\zeta=0$ at the maximum cross-coupling condition, whereas $0<\zeta \leqslant 1$ in the electric-only case.

The resemblance of band structures traces back to the fact that for the fully coupled system, the band structure expressed as a shift $\Delta \omega\left(k_{\|}\right)=\omega\left(k_{\|}\right)-\omega_{0}$ is closely connected to the sum of the band structures of the uncoupled electric and magnetic band in Fig 3(d). In Fig. 3(f), the sum of frequency shifts for the two bands of the uncoupled system $\Delta \omega_{E}\left(k_{\|}\right)+$ $\Delta \omega_{H}\left(k_{\|}\right)+\omega_{0}$ is overplotted with the fully coupled result. Since the magnetic mode is almost flat, the result is that the fully coupled system closely resembles the purely electric system in band structure. The mathematical reasoning behind this summation argument traces back to analysis of the matrix form of the bare polarizability Eq. (4). As cross coupling approaches the maximally coupled case, one eigenvalue vanishes while the second eigenvalue simplifies to the sum of the diagonal contributions. Similar reasoning extends to the full effective polarizability that includes the lattice summation. Thereby, one dispersion band, corresponding to the vanishing eigenpolarizability, converges to $\Delta \omega=0$ and vanishes in strength, while the second band approaches the sum of the dispersions in the uncoupled systems.

\section{CONCLUSION}

To conclude, we discussed a method based on magnetoelectric point-dipole interactions and Ewald lattice summation to approximate the dispersion relation of two-dimensional lattices of bianisotropic scatterers, accounting for all retarded electrodynamic interparticle interactions. Our results show that simple square lattices of plasmon rods that are dense, i.e., of subdiffraction pitch, support a mode structure characterized by weakly confined guided modes with a dispersion very close to the light line for frequencies to the red of the single scatterer resonance, and tightly confined guided modes at wave vectors well away from the light line. These modes are dispersive in a manner similar to results obtained previously for 1D plasmon chains, with the added complication that modes can have a mixed transverse and longitudinal character. Lattices of scatterers that have an intrinsically decoupled electric and magnetic polarizability in each element will have a dispersion in which modes have a mixed magnetoelectric character. Furthermore, we reported how introduction of bianisotropy in each building block modifies the dispersion. For full cross coupling, a single mode prevails with the electric and magnetic dipoles being interlocked with equal magnitude and a fixed $\pi / 2$ phase.

As outlook, while we presented results for simple square lattices of split ring type resonators with just an in-plane electric moment and out-of-plane magnetic moment, the method is easily generalized to deal with arbitrarily complex multielement lattices of arbitrary magnetoelectric scatterers, provided that the dipole approximation is met. Thus, our method is important for many structures, including metasurface designs with complex unit cells that comprise many elements. Since the method is fast, it should thus be possible to screen many different lattice symmetries and arrangements in the unit cell for desirable properties, such as minimized bianisotropy and spatial dispersion. As regards actual measurements of such dispersion relations, we note that measurements in the visible domain would likely be hampered by the much stronger Ohmic damping than assumed in this work, and the drawback that the Brillouin zone extends to very large wave vectors, rendering even near-field microscopy impractical. These are exactly the drawbacks that have made it impossible to verify the projected dispersion of 1D plasmon chains beyond the light line $[14,15]$. However, in the radiofrequency domain, 
the dispersion relations should be more readily available. The rf domain offers as advantages that low-loss split rings can be made, that near-field probes with $\lambda / 100$ resolution are routine, and that both phase and amplitude can be mapped so that $k$ is directly measured. Finally, we believe that the calculated dispersion relations should be important for lasing spaser experiments, in which lattices are studied in presence of gain to achieve lasing. The modes with the best tradeoff between loss and confinement should be found close to and just below the light line, rather than at the $k_{\|}=0$ point, which was proposed as the lasing mode originally [28]. Furthermore, from work on Yagi-Uda antennas in the optical domain, it is well known that directional scattering and directional emission of embedded emitters is strongly linked to the modes of 1D particle chains just below the light line [53-56]. Similarly, for 2D arrays our work points at design strategies for shaping directional emission.

\section{ACKNOWLEDGMENTS}

We are grateful to Y. Chen and L. Langguth for insightful comments. This work is part of the research program of the Stichting voor Fundamenteel Onderzoek der Materie (FOM), which is financially supported by the Nederlandse Organisatie voor Wetenschappelijk Onderzoek (NWO). A.F.K. gratefully acknowledges a NWO-VIDI grant for financial support. P.L. acknowledges support by the Carlsberg Foundation as well as the Danish Agency for Science, Technology and Innovation (Det Frie Forskningsråd) (Grant No. 11-116740).
[1] R. Wood, Phys. Rev. 48, 928 (1935).

[2] U. Fano, J. Opt. Soc. Am. 31, 213 (1941).

[3] E. G. Loewen and E. Popov, in Diffraction Gratings and Applications, edited by B. J. Thompson (CRC Press, New York, USA, 1997).

[4] E. Yablonovitch, Phys. Rev. Lett. 58, 2059 (1987).

[5] J. D. Joannopoulos, P. R. Villeneuve, and S. Fan, Nature (London) 386, 143 (1997).

[6] S. Linden, J. Kuhl, and H. Giessen, Phys. Rev. Lett. 86, 4688 (2001).

[7] N. I. Zheludev and Y. S. Kivshar, Nat. Mater. 11, 917 (2012).

[8] A. V. Kildishev, A. Boltasseva, and V. M. Shalaev, Science 339, 1232009 (2013).

[9] M. Quinten, A. Leitner, J. R. Krenn, and F. R. Aussenegg, Opt. Lett. 23, 1331 (1998).

[10] S. A. Maier, P. G. Kik, H. A. Atwater, S. Meltzer, E. Harel, B. E. Koel, and A. A. G. Requicha, Nat. Mater. 2, 229 (2003).

[11] A. F. Koenderink and A. Polman, Phys. Rev. B 74, 033402 (2006).

[12] M. L. Brongersma, J. W. Hartman, and H. A. Atwater, Phys. Rev. B 62, R16356 (2000).

[13] W. H. Weber and G. W. Ford, Phys. Rev. B 70, 125429 (2004).

[14] R. de Waele, A. F. Koenderink, and A. Polman, Nano Lett. 7, 2004 (2007).

[15] T. Yang and K. B. Crozier, Opt. Express 16, 8570 (2008).

[16] A. L. Fructos, S. Campione, F. Capolino, and F. Mesa, J. Opt. Soc. Am. B 28, 1446 (2011).

[17] C. M. Linton, SIAM Rev. 52, 630 (2010).

[18] G. Vecchi, V. Giannini, and J. Gómez Rivas, Phys. Rev. B 80, 201401 (2009).

[19] G. Vecchi, V. Giannini, and J. Gómez Rivas, Phys. Rev. Lett. 102, 146807 (2009).

[20] B. Auguié and W. L. Barnes, Phys. Rev. Lett. 101, 143902 (2008).

[21] V. G. Kravets, F. Schedin, and A. N. Grigorenko, Phys. Rev. Lett. 101, 087403 (2008).

[22] R. F. Aroca, Phys. Chem. Chem. Phys. 15, 5355 (2013).

[23] L. Langguth and A. F. Koenderink, Opt. Express 22, 15397 (2014).

[24] J. N. Anker, W. P. Hall, O. Lyandres, N. C. Shah, J. Zhao, and R. P. Van Duyne, Nat. Mater. 7, 442 (2008).
[25] S. S. Aćimović, M. P. Kreuzer, M. U. González, and R. Quidant, ACS Nano 3, 1231 (2009).

[26] S. R. K. Rodriguez, S. Murai, M. A. Verschuuren, and J. G. Rivas, Phys. Rev. Lett. 109, 166803 (2012).

[27] G. Lozano, D. J. Louwers, S. R. Rodríguez, S. Murai, O. T. A. Jansen, M. A. Verschuuren, and J. Gómez Rivas, Light Sci. Appl. 2, e66 (2013).

[28] N. I. Zheludev, S. L. Prosvirnin, N. Papasimakis, and V. A. Fedotov, Nat. Photonics 2, 351 (2008).

[29] A. Polman, Science 322, 868 (2008).

[30] P. Lunnemann, I. Sersic, and A. F. Koenderink, Phys. Rev. B 88, 245109 (2013).

[31] I. Sersic, M. Frimmer, E. Verhagen, and A. F. Koenderink, Phys. Rev. Lett. 103, 213902 (2009).

[32] C. Enkrich, M. Wegener, S. Linden, S. Burger, L. Zschiedrich, F. Schmidt, J. F. Zhou, T. Koschny, and C. M. Soukoulis, Phys. Rev. Lett. 95, 203901 (2005).

[33] C. Rockstuhl, T. Zentgraf, H. Guo, N. Liu, C. Etrich, I. Loa, K. Syassen, J. Kuhl, F. Lederer, and H. Giessen, Appl. Phys. B 84, 219 (2006).

[34] C. R. Simovski and S. A. Tretyakov, Phys. Rev. B 75, 195111 (2007).

[35] C. Menzel, C. Rockstuhl, T. Paul, F. Lederer, and T. Pertsch, Phys. Rev. B 77, 195328 (2008).

[36] A. Alù, Phys. Rev. B 83, 081102 (2011).

[37] Y.-R. Zhen, K. H. Fung, and C. T. Chan, Phys. Rev. B 78, 035419 (2008).

[38] I. Lindell, A. Sihvola, S. Tretyakov, and A. Viitanen, Electromagnetic Waves in Chiral and Bi-Isotropic Media (Artech Print on Demand, Norwood, USA, 1994), p. 352.

[39] I. Sersic, C. Tuambilangana, T. Kampfrath, and A. F. Koenderink, Phys. Rev. B 83, 245102 (2011).

[40] I. Sersic, M. A. van de Haar, F. B. Arango, and A. F. Koenderink, Phys. Rev. Lett. 108, 223903 (2012).

[41] P. Belov, S. Maslovski, K. Simovski, and S. Tretyakov, Tech. Phys. Lett. 29, 718 (2003).

[42] B. T. Draine and P. J. Flatau, J. Opt. Soc. Am. A 11, 1491 (1994).

[43] F. J. García de Abajo, Rev. Mod. Phys. 79, 1267 (2007).

[44] A. Kwadrin and A. F. Koenderink, Phys. Rev. B 89, 045120 (2014). 
[45] A. S. Barker and R. Loudon, Rev. Mod. Phys. 44, 18 (1972).

[46] P. B. Johnson and R. W. Christy, Phys. Rev. B 6, 4370 (1972).

[47] M. Husnik, M. W. Klein, N. Feth, J. Niegemann, K. Busch, S. Linden, and M. Wegener, Nat. Photonics 2, 614 (2008).

[48] E. Prodan, C. Radloff, N. J. Halas, and P. Nordlander, Science 302, 419 (2003).

[49] N. Liu, H. Liu, S. Zhu, and H. Giessen, Nat. Photonics 3, 157 (2009).

[50] C. F. Bohren and D. R. Huffman, Absorption and Scattering of Light by Small Particles (Wiley, New York, 1983).
[51] A. E. Krasnok, A. E. Miroshnichenko, P. A. Belov, and Y. S. Kivshar, Opt. Express 20, 20599 (2012).

[52] F. B. Arango and A. F. Koenderink, New J. Phys. 15, 073023 (2013).

[53] T. Kosako, Y. Kadoya, and H. F. Hofmann, Nat. Photonics 4, 312 (2010).

[54] A. F. Koenderink, Nano Lett. 9, 4228 (2009).

[55] F. Bernal Arango, A. Kwadrin, and A. F. Koenderink, ACS Nano 6, 10156 (2012).

[56] A. G. Curto, G. Volpe, T. H. Taminiau, M. P. Kreuzer, R. Quidant, and N. F. van Hulst, Science 329, 930 (2010). 